\title{
A Dual Layer Frequency Selective Surface Reflector for Wideband Applications
}

\author{
Ayan CHATTERJEE, Susanta Kumar PARUI \\ Dept. of Electronics \& Tele-Comm. Engineering, Indian Institute of Engineering Science \& Technology, \\ Shibpur, Howrah-711103, West Bengal, India \\ ayanmrinal26@gmail.com, arkapv@yahoo.com
}

Manuscript received October 9, 2015

\begin{abstract}
A dual-layer, bandstop frequency selective surface (FSS) is presented in this paper for wideband applications. Each layer uses patch type FSS with slots for miniaturization and are cascaded with an air gap in between. The low-profile FSS with unit cell dimension on the order of $0.2 \lambda_{0} \times 0.2 \lambda_{0}$ provides transmission coefficient below $-10 \mathrm{~dB}$ in the frequency range of $4-7 \mathrm{GHz}$ with $56 \%$ bandwidth. The FSS exhibits a nearly linear phase variation with frequency in the operating band and can be used as a substrate below planar wide band antennas with bidirectional radiation for enhancing its gain, directivity in the broadside direction as well as shielding it against nearby conductive surfaces such as metal cases, other printed antennas. Detailed design method, equivalent circuit analysis and measurement results of the FSS are presented in this paper.
\end{abstract}

\section{Keywords}

Frequency Selective Surface, reflection phase, bandstop, wideband, reflector

\section{Introduction}

Frequency Selective Surfaces (FSSs) are wireless counterpart of traditional filters that are used in radio frequency (RF) circuits. FSSs are periodic arrays of metallic patch or apertures on a dielectric slab and are used for fully reflecting (patch) or transmitting (apertures) radio waves from antenna at specific frequencies or band of frequencies [1]. Since introduction, FSS is being used in radome, subreflector, polarizer, satellite transponder etc. [1-3]. In recent years, researchers have become interested in designing wideband FSS structures of both bandpass and bandstop response for their applications in gain or directivity enhancement of broadband, omni-directional antennas such as slot antennas when used as their substrates (bandstop) or superstrates (bandpass) [4-9]. Such enhancements are also possible with simple metallic reflectors placed at quarter wavelength spacing from the antenna, but their functioning is optimal over a smaller bandwidth. Moreover FSS also provides shielding to the antenna against nearby conductive elements [6]. Gain and bandwidth enhancement of a dual band $U$ slot patch antenna using loop shaped FSS as substrate was proposed by Hsing-Yi Chen and Yu Tao [5]. A high gain edge fed patch antenna was designed by W. Yang et al. [9] where gain of the antenna and its array was enhanced over a wide bandwidth using artificial magnetic conductors (AMC).

In general multiple FSS layers are cascaded to achieve multiband or broadband response [10-13]. FSS layers with distant frequency bands are used to achieve multiband response [10] whereas those with closely spaced resonating frequencies lead to higher order wideband response. In case of wideband FSS, introduction of air gap between the layers reduce their mutual coupling leading to a significant reflection over the bandwidth. Cascaded FSS screen with a nearly linear variation of transmission or reflection phase over a wide bandwidth is very useful in enhancing radiation properties of antennas with bidirectional radiation such as wide slot antennas as was discussed in [6]

In this paper, the design of a dual-layer, reflective FSS is proposed. The proposed FSS gives a transmission coefficient below $-10 \mathrm{~dB}$ over a wide frequency range of 4-7 GHz and thus works as a good reflector offering $56 \%$ bandwidth. The FSS layers have been simulated with ANSYS HFSS for different parameters. Performance of the cascaded FSS has been experimentally verified. An equivalent circuit model of the FSS is also presented and analyzed using ADS simulator. The FSS can be used as a reflector with almost linear phase variation with frequency in any wideband antenna as a substrate for its performance improvement and at the same time shielding the antenna from any nearby conductive element.

\section{Design of the FSS}

A three dimensional view of the proposed dual-layer FSS with complete geometry is shown in Fig. 1(a). The proposed FSS is composed of two patch type layers cascaded with air gap in between. Patch type FSS is chosen to achieve the response of a bandstop filter and thus good reflection characteristics in the frequency band of 4-7 GHz. In layer I, a square patch of $10 \mathrm{~mm} \times 10 \mathrm{~mm}$ is used with 


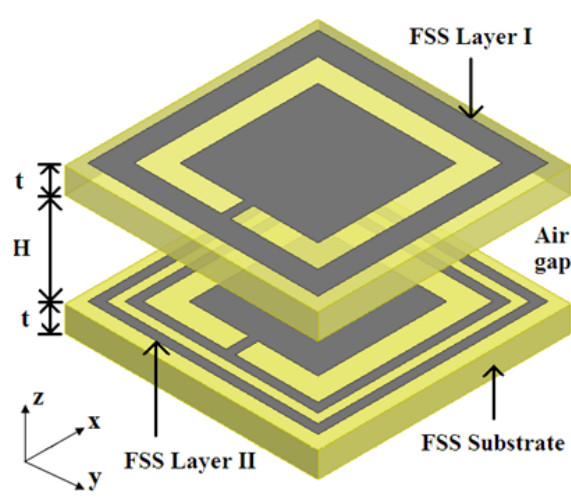

(a)

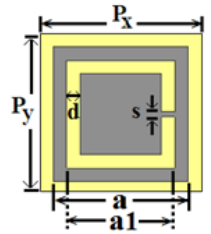

(b)

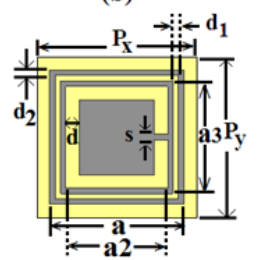

(c)
Fig. 1. (a) 3-D view of the cascaded FSS. Unit cell of the schematic of the (b) FSS Layer I (c) FSS Layer II (all dimensions are in $\mathrm{mm}$ ).

\begin{tabular}{|c|c|c|c|c|c|c|c|c|c|}
\hline$P_{\mathrm{x}}=P_{\mathrm{y}}$ & $a$ & $a_{1}$ & $a_{2}$ & $a_{3}$ & $d$ & $d_{1}$ & $d_{2}$ & $g$ & $s$ \\
\hline 11 & 10 & 8 & 7.6 & 8.4 & 1 & 0.4 & 0.4 & 0.4 & 1 \\
\hline
\end{tabular}

Tab. 1. Unit cell dimensions of FSS layers (in $\mathrm{mm}$ ).

a slot cut on it with the shape of a split ring to cover 5-6 GHz band with resonance at $5.5 \mathrm{GHz}$. Unit cell geometry of the FSS layer I is shown in Fig. 1(b). The slot is cut to reduce resonating frequency of the patch (discussed in the next section) as because without a slot it resonates at a much higher frequency. The slot length $a_{1}$ is determined by the following equation [2]:

$$
\text { Slot length }=\frac{c_{0}}{4 \cdot f \cdot \sqrt{\left(\varepsilon_{r}+1\right) / 2}}
$$

where $\mathrm{c}_{0}$ stands for velocity of light in free space $\left(3 \times 10^{8} \mathrm{~m} / \mathrm{s}\right), f$ is the resonating frequency $(5.5 \mathrm{GHz})$ and $\varepsilon_{\mathrm{r}}$ is relative permittivity of the dielectric used and in the proposed design FR4 (glass-epoxy) is chosen as substrate in both the layers and $\varepsilon_{\mathrm{r}}=4.4$. The substrate thickness is $t=1.2 \mathrm{~mm}$. The FSS layer II whose unit cell is shown in Fig. 1(c) is designed to achieve two stop bands covering 4-5 GHz and 6-7 GHz and resonances near $4.5 \mathrm{GHz}$ and $6.5 \mathrm{GHz}$, respectively. For $6-7 \mathrm{GHz}$ a similar design of patch is chosen as layer I and the slot length $a_{2}$ is calculated using (1) for $6.5 \mathrm{GHz}$. A square loop shaped patch is chosen for the $4-5 \mathrm{GHz}$ band and is located surrounding the square patch. Length of the square loop $a$ is also selected using (1) for $4.5 \mathrm{GHz}$. After obtaining the values, a detailed parametric analysis was carried out (discussed in the next section) to obtain the desired response and the dimensions of unit cells of FSS layer I and II are given in Tab. 1. It can be seen, horizontal and vertical periodicities of unit cells i.e. $P_{\mathrm{x}}$ and $P_{\mathrm{y}}$ for both the layers are kept same and $P_{\mathrm{x}}=P_{\mathrm{y}}$. An air gap of $H \mathrm{~mm}$ is maintained between the layers of FSS to achieve the overall transmission coefficient well below $-10 \mathrm{~dB}$ and to achieve an almost linear variation of reflection phase with frequency in 4-7 GHz.

\section{Characteristics of the FSS Layers and Parametric Analysis}

The full wave EM simulation of individual FSS layers and the cascaded FSS has been carried out using finite element method based ANSYS HFSS to calculate their transmission coefficients and reflection phase response. During simulation the FSS has been assumed as infinite periodic structure and four sides of the unit cell are set to periodic boundary conditions (PBC). Finally the structure is excited with a plane wave using Floquet ports with the electric field (E) oriented along Y axis (Fig. 1) that is vertical polarization. Normalized transmission coefficient $(\mathrm{dB})$ for the FSS layer I is shown in Fig. 2(a) for different values of the slot gap $s$. The study shows that with reduction in the gap, the resonating frequency shifts downward. With a reduction in gap $s$, the current path length in the patch increases as can be seen from surface current distribution in Fig. 3(a) and this increases effective wavelength that led to a reduced resonating frequency.

For the FSS layer II, normalized transmission coefficient $(\mathrm{dB})$ for different values of spacing $d_{1}$ between the patch and loop is shown in Fig. 2(b). As can be observed

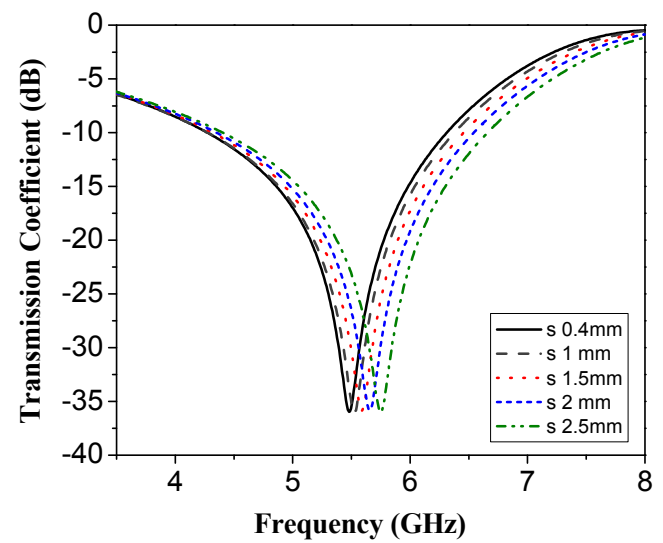

(a)

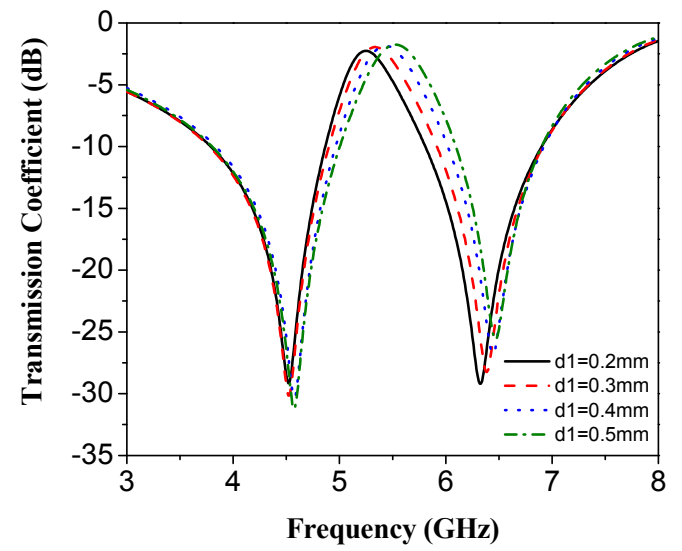

(b)

Fig. 2. Simulated transmission coefficient with parametric study of the (a) FSS layer I (for different $s \mathrm{~mm}$ ), (b) FSS Layer II (for different $d_{1} \mathrm{~mm}$ ). 


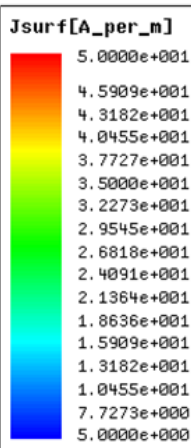

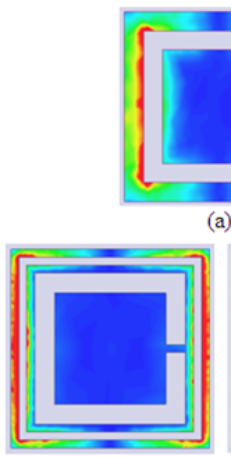

(b)

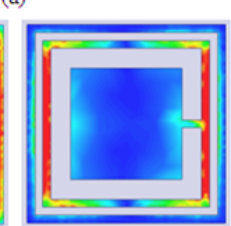

(c)
Fig. 3. Surface current $(\mathrm{A} / \mathrm{m})$ on FSS (a) Layer I at $5.48 \mathrm{GHz}$, Layer II at (b) $4.58 \mathrm{GHz}$ and (c) $6.45 \mathrm{GHz}$.

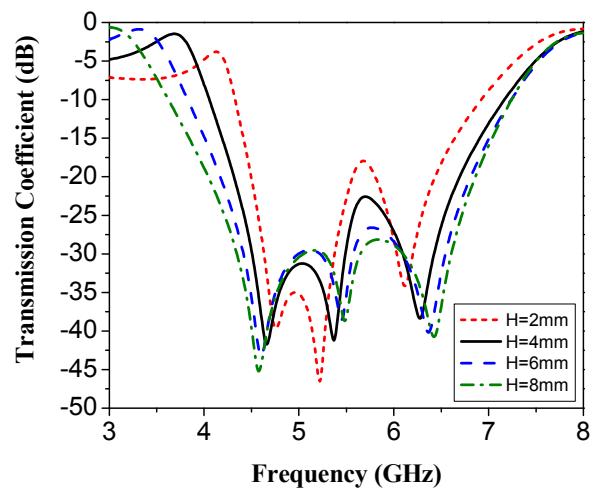

(a)

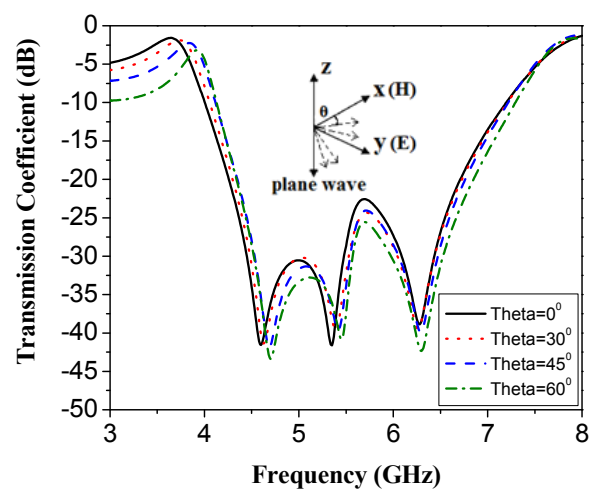

(b)

Fig. 4. Simulated transmission coefficient of the cascaded FSS for (a) different gap $H$, (b) different incidence angles of plane wave and $H=4 \mathrm{~mm}$.

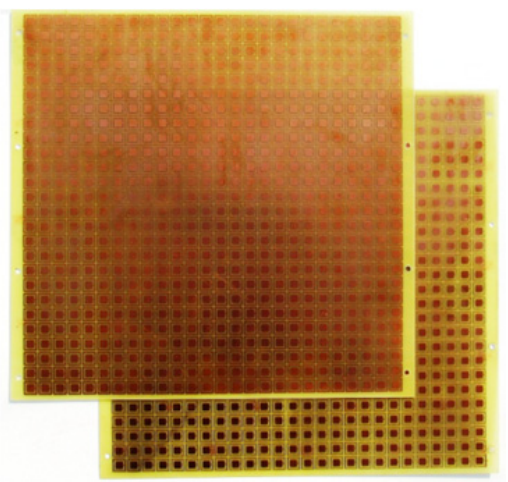

Fig. 5. Fabricated prototype of the cascaded FSS (676 elements each). from Fig. 2(b), the FSS layer II is a dual band FSS with their resonances near $4.5 \mathrm{GHz}$ and $6.5 \mathrm{GHz}$. The variation of spacing $d_{1}$ does not have significant impact on the transmission coefficient except a very small shift in the operating frequency band for the square loop shaped FSS. Surface current on unit cell of the FSS layer II shows that at $4.58 \mathrm{GHz}$ the current mainly flows on the square loop FSS whereas at $6.45 \mathrm{GHz}$ significant current flows around the slot on the square patch.

Simulation results for transmission coefficient of the cascaded FSS is shown in Fig. 4(a) for different values of the air gap $H \mathrm{~mm}$ and dimensions as mentioned in Tab. 1. All simulations are carried out for normal incidence of plane wave. The air gap is used to reduce the coupling between two FSS layers and to keep the transmission level well below $-10 \mathrm{~dB}$. As can be observed from Fig. 4(a) increasing air gap reduces the transmission as well as increases the bandwidth. The dual layer FSS is then simulated for different incidence angles of plane wave theta $(\theta)$ in the TE (Transverse Electric) plane keeping $H=4 \mathrm{~mm}$. As can be seen from Fig. 4(b) transmission response of the proposed FSS is not significantly affected with change in angle of incidence even upto $60^{\circ}$.

A fabricated prototype of the cascaded FSS is shown in Fig. 5 for $H=4 \mathrm{~mm}$ with 676 elements each having dimensions as mentioned in Tab. 1. Transmission response of the FSS is measured with a signal generator, two standard gain horn antennas with the FSS placed in between and a power meter. Simulation and measurement results for $H=4 \mathrm{~mm}$ is given in Fig. 6(a) which shows $56 \%$ measured

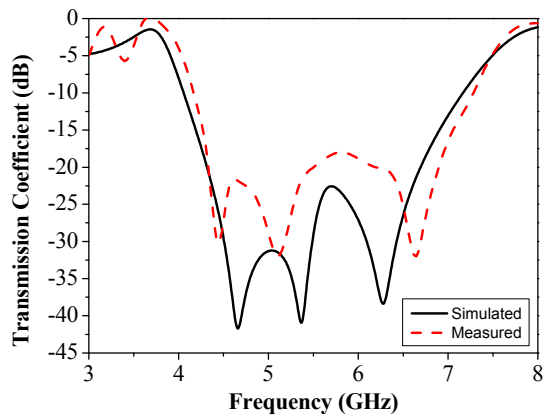

(a)

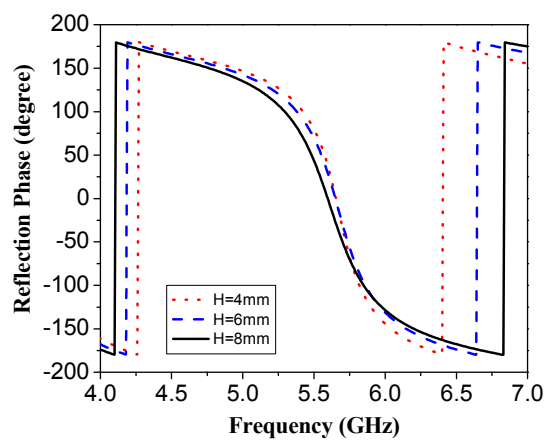

(b)

Fig. 6. (a) Simulation and measurement results for transmission coefficient of cascaded FSS of dimensions mentioned in Tab. 1 and $H=4 \mathrm{~mm}$. (b) Reflection phase response of the cascaded FSS for different $H \mathrm{~mm}$. 
transmission bandwidth $(-10 \mathrm{~dB})$ with respect to centre frequency in comparison to a simulated value of $54 \%$. The proposed design also gives a nearly linear variation of reflection phase with frequency over the band of interest as shown in Fig. 6(b) for different values of air gap $H$ and it can be observed that increasing air gap increases this bandwidth. Such variation of phase makes the FSS useful for gain and directivity enhancement of antennas with bidirectional or omni-directional radiation over a wide frequency band [6].

\section{Equivalent Circuit Model}

An equivalent circuit for unit cell of the dual layer FSS is shown in Fig. 7. The patch with slot in the layer I is modeled as a series combination of inductor $\mathrm{L}_{1}$ and a capacitor $\mathrm{C}_{1}$ whereas a parallel combination of two such LC resonators $\mathrm{L}_{2}, \mathrm{C}_{2}$ and $\mathrm{L}_{3}, \mathrm{C}_{3}$ represents the layer II for slot loaded patch and loop shaped FSSs respectively. All the FSS structures are modeled as series LC resonators as all of them are patch type FSS and exhibit bandstop filter response [1].

As can be seen from Fig. 3, for the two slot loaded patch type FSSs in the layer I and II, the region beside slot in the patch allowing current to flow accounts for the inductors $\mathrm{L}_{1}, \mathrm{~L}_{2}$ and the gap between two successive patches accounts for the capacitors $\mathrm{C}_{1}, \mathrm{C}_{2}$. Similarly, for the loop shaped patch, the two sides of the loop through which the induced current flows as shown in Fig. 3(b), represents the inductor $\mathrm{L}_{3}$ and the gap between two consecutive loops along which the electric field is oriented, accounts for the capacitor $\mathrm{C}_{3}$. These $\mathrm{LC}$ values can be calculated using the following equations (2) and (3) as presented in [14].

$$
\begin{gathered}
C=\varepsilon_{0} \varepsilon_{\text {eff }} \frac{2 P}{\pi} \ln \left(\frac{1}{\sin \frac{\pi m}{2 P}}\right), \\
L=\mu_{0} \mu_{\text {eff }} \frac{b}{2 \pi} \ln \left(\frac{1}{\sin \frac{\pi w}{2 b}}\right) .
\end{gathered}
$$

Here $m$ is spacing between consecutive patches, $\varepsilon_{0}$ (free space permittivity) $=8.85 \times 10^{-12} \mathrm{~F} / \mathrm{m}, \varepsilon_{\text {eff }}$ (effective permittivity $)=\left(\varepsilon_{\mathrm{r}}+1\right) / 2=2.7$ and $P$ is periodicity of the patch whereas $\mu_{0}$ (free space permeability) $=4 \pi \times 10^{-7} \mathrm{H} / \mathrm{m}, \mu_{\text {eff }}$ (effective permeability) $=\left(\mu_{\mathrm{r}}+1\right) / 2=1, b$ is length of the inductors, i.e. length of the slots and $w$ is width of the inductors. The above mentioned parameters required to calculate the LC values corresponding to the patches in both the layers are given in Tab. 2 in terms of unit cell dimensions (given in Tab. 1).

\begin{tabular}{|c|c|c|c|}
\hline Parameter & Layer I patch & Layer II patch & Layer II loop \\
\hline$P$ & $P_{\mathrm{x}}=P_{\mathrm{y}}=11 \mathrm{~mm}$ & $P_{\mathrm{x}}=P_{\mathrm{y}}=11 \mathrm{~mm}$ & $P_{\mathrm{x}}=P_{\mathrm{y}}=11 \mathrm{~mm}$ \\
\hline$m$ & $P-a=1 \mathrm{~mm}$ & $P-a_{3}=2.6 \mathrm{~mm}$ & $P-a=1 \mathrm{~mm}$ \\
\hline$w$ & $\left(a-a_{1}\right) / 2=1 \mathrm{~mm}$ & $\left(a_{3}-a_{2}\right) / 2=0.4 \mathrm{~mm}$ & $2 d_{2}$ \\
\hline$b$ & $a_{1}=8 \mathrm{~mm}$ & $a_{2}=7.6 \mathrm{~mm}$ & $P_{\mathrm{x}}=P_{\mathrm{y}}=11 \mathrm{~mm}$ \\
\hline
\end{tabular}

Tab. 2. Parameters used for LC calculation.

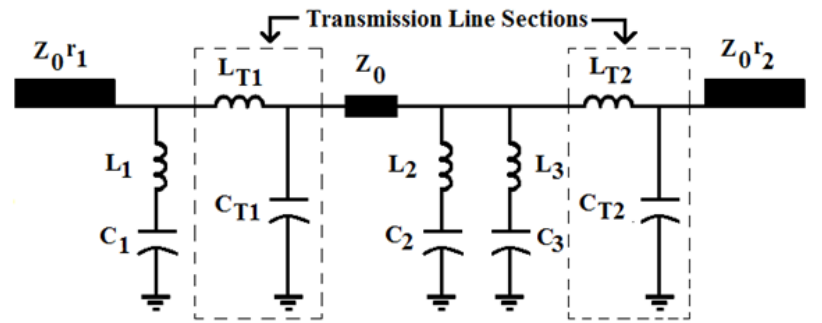

Fig. 7. Simple equivalent circuit model for FSS unit cell.

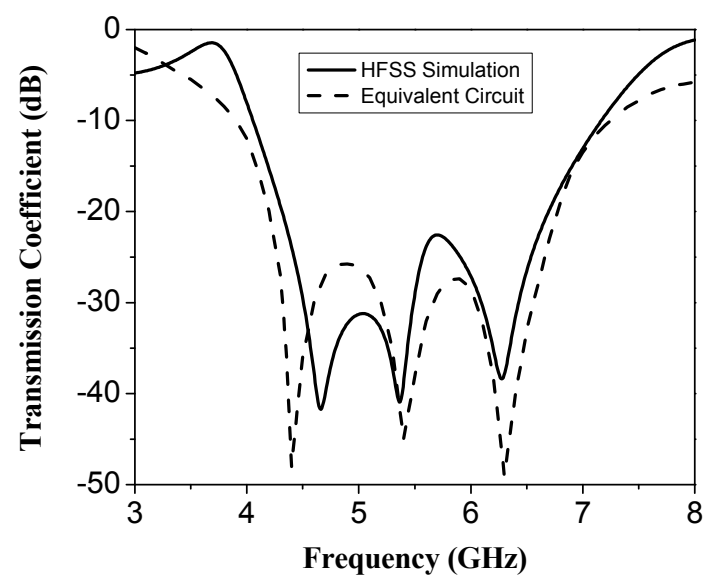

Fig. 8. Transmission coefficient of the FSS using HFSS and circuit model.

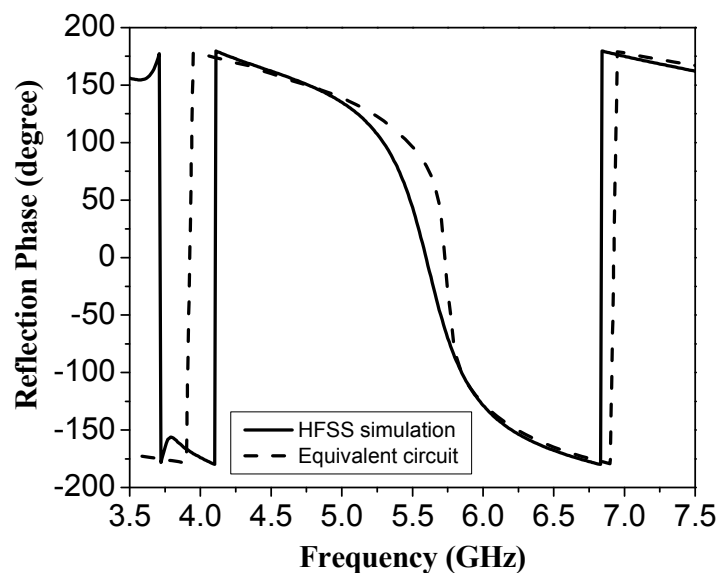

Fig. 9. Reflection phase of the FSS using HFSS and circuit model.

It can be observed from Tab. 2 that for $\mathrm{L}_{3}$ corresponding to square loop FSS, width of the inductive strip is taken twice the width $d_{2}$ and the reason is in an array of square loop FSS, for the induced electric field along two closely spaced sides of the loop, effective width of the inductive strips become $2 d_{2}$ as mentioned in [15-17].

In the circuit, the dielectric substrates in FSS layer I and II are modeled as transmission line sections with series inductors $\mathrm{L}_{\mathrm{T} 1}, \mathrm{~L}_{\mathrm{T} 2}$ and shunt capacitors $\mathrm{C}_{\mathrm{T} 1}, \mathrm{C}_{\mathrm{T} 2}$. Characteristic impedance of this transmission line is assumed to be $Z_{0} / \sqrt{ } \varepsilon_{\mathrm{r}}$ where $\varepsilon_{\mathrm{r}}$ is relative permittivity of the dielectric (4.4 for FR4) and $Z_{0}$ is free space wave impedance ( 377 ohms). The values of $L_{T}$ and $C_{T}$ can be calculated from the Telegrapher's model for TEM transmission lines using following equations (4) and (5). 


$$
\begin{aligned}
& L_{\mathrm{T} 1}=L_{\mathrm{T} 2}=\mu_{0} \mu_{\mathrm{r}} t, \\
& C_{\mathrm{T} 1}=C_{\mathrm{T} 2}=\varepsilon_{0} \varepsilon_{\mathrm{r}} t .
\end{aligned}
$$

Here $t$ is substrate thickness $=1.2 \mathrm{~mm}$ for both the layers. Free spaces on both ends of the cascaded FSS are modeled here as semi-infinite transmission lines with characteristic impedances of $Z_{0} r_{1}$ and $\mathrm{Z}_{0} r_{2}$ respectively where $r_{1}$ and $r_{2}$ are the normalized source and load impedances and for free space $r_{1}=r_{2}=1$. The air gap between FSS layers is also represented by $\mathrm{Z}_{0}$.

\begin{tabular}{|c|c|c|c|c|c|c|c|}
\hline$L_{1}$ & $C_{1}$ & $L_{2}$ & $C_{2}$ & $L_{3}$ & $C_{3}$ & $L_{\mathrm{T} 1}=L_{\mathrm{T} 2}$ & $C_{\mathrm{T} 1}=C_{\mathrm{T} 2}$ \\
\hline 2.62 & 0.326 & 3.79 & 0.169 & 4.7 & 0.326 & 1.51 & 46.728 \\
$\mathrm{nH}$ & $\mathrm{pF}$ & $\mathrm{nH}$ & $\mathrm{pF}$ & $\mathrm{nH}$ & $\mathrm{pF}$ & $\mathrm{nH}$ & $\mathrm{fF}$ \\
\hline
\end{tabular}

Tab. 3. Inductor and capacitor values for the circuit.

The proposed equivalent circuit was simulated using ADS (Advanced Design System) with the LC values provided in Tab. 3 for transmission coefficient and the result is shown in Fig. 8 for comparison with the results obtained from HFSS simulation. It can be seen that circuit simulation matches well with the EM simulation except a little difference in the lowest frequency corresponding to square loop in layer II. While estimating the value of $\mathrm{L}_{3}$, two closely spaced adjacent sides of the loops have been considered to contribute for the inductance and the width is taken as $2 d_{2}$ neglecting the spacing between them [15-17]. This approximation accounts for the difference in lowest resonating frequency. Moreover, changing the air gap $H$ between two FSS layers is difficult to model in the equivalent circuit which also accounts for the difference in transmission response achieved from circuit model and HFSS simulation. Reflection phase response obtained from EM simulation and circuit model analysis is shown in Fig. 9 for comparison and they matches well each other only with a small change due to the reasons stated above.

\begin{tabular}{|c|c|c|c|}
\hline Parameter & Ref[18] & Ref[19] & Proposed \\
\hline Frequency band & $8-13 \mathrm{GHz}$ & $8-11 \mathrm{GHz}$ & $4-7 \mathrm{GHz}$ \\
\hline$-10 \mathrm{~dB}$ bandwidth & $45 \%$ & $32 \%$ & $54 \%$ \\
\hline Number of layers & 1 & 2 & 2 \\
\hline Layer spacing (mm) & NA & $7.43\left(\lambda_{0} / 4\right)$ & $4\left(\lambda_{0} / 13\right)$ \\
\hline Unit cell (mm) & $20 \times 20$ & $13 \times 13$ & $11 \times 11$ \\
\hline Angular stability & $30^{\circ}$ & - & $60^{\circ}$ \\
\hline Reflection phase & - & - & Linear \\
\hline Technology used & Fractal & Cascading & Cascading \\
\hline
\end{tabular}

Tab. 4. Comparison of the proposed design with previous designs.

A comparison of the proposed design and its performance with some previous designs [18], [19] is presented in Tab. 4. It can be seen that the proposed design is compact and gives a wider bandwidth than the structures in [18] and [19] but with two layers of FSS unlike that in [18]. The proposed FSS shows better angular stability than [18] though it is polarization dependent and in terms of spacing between the layers it is low profile in comparison to [19].
Novelty of the proposed FSS is its compactness $\left(0.2 \lambda_{0} \times 0.2 \lambda_{0}\right.$ for the center frequency), low profile $\left(0.1 \lambda_{0}\right)$ with better angular stability upto $60^{\circ}$ and almost linear variation of reflection phase with frequency in the desired band.

\section{Conclusions}

In this paper, design procedure and analysis of a broadband, reflective FSS operating in the 4-7 GHz band is presented. The FSS is composed of two layers with substrate thickness of $0.02 \lambda_{0}$ and an air gap of $0.1 \lambda_{0}\left(\lambda_{0}\right.$ is the wavelength corresponding to the center frequency of $5.5 \mathrm{GHz}$ ) leading to a low profile design. The proposed design provides $-10 \mathrm{~dB}$ bandwidth of $56 \%$ with high reflectivity as well as stable performance for different angles of incidence. An equivalent circuit model for the FSS is presented and analyzed which matches well with the EM simulation result. Measurement result for the FSS is given in the paper that confirms the simulated one. The proposed FSS gives a nearly linear phase variation over the frequency band which makes it useful for enhancing radiation properties such as gain, directivity, front to back ratio of the antennas with omni-directional or bi-directional radiation (slot antennas, CPW antenna) over a wide bandwidth when used as a reflector.

\section{Acknowledgments}

The authors would like to acknowledge CSIR, India (award no. 08/003(0101)/2014-EMR-I) for funding. The authors are also grateful to Dr. P. P. Sarkar, DETS, University of Kalyani, India for the measurements.

\section{References}

[1] MUNK, B.A. Frequency Selective Surfaces-Theory and Design. New York: Wiley, 2000. DOI: 10.1002/0471723770

[2] BAYATPUR, F. Metamaterial Inspired Frequency Selective Surfaces: A dissertation submitted in the partial fulfillment of the requirements for the degree of Doctor of Philosophy, University of Michigan, 2009. DOI: 10.1.1.402.3931

[3] LAZARO, A., LORENZO, J., VILlARINO, R., GIRBAU, D. Backscatter transponder based on frequency selective surface for FMCW radar applications. Radioengineering, 2014, vol. 23, no. 2, p. 632-641. DOI: $10.13164 /$ re.2014.0632

[4] PIRHADI, A., BAHRAMI, H., NASRI, J. Wideband high directive aperture coupled microstrip antenna design by using a FSS superstrate layer. IEEE Transactions on Antennas and Propagation, 2012, vol. 60, no. 4, p. 2101-2106. DOI: 10.1109/ TAP.2012.2186230

[5] CHEN, H-Y., TAO. Y. Performance improvement of a U-slot patch antenna using a dual-band frequency selective surface with modified Jerusalem cross elements. IEEE Transactions on Antennas and Propagation, 2011, vol. 59, no. 9, p. 3482-3486. DOI: 10.1109/TAP.2011.2161440

[6] CHATTERJEE, A., PARUI, S.K. Gain Enhancement of a wide slot antenna using a second-order bandpass frequency selective 
surface. Radioengineering, 2015, vol. 24, no. 2, p. 455-461. DOI: $10.13164 /$ re.2015.0455

[7] LEE, Y.J., YEO, J., MITTRA, R., PARK, W.S. Design of a frequency selective surface (FSS) type superstrate for dual-band directivity enhancement of microstrip patch antennas. In IEEE Antennas and Propagation Society International Symposium, 2005, vol. 3A, p. 2-5. DOI: 10.1109/APS.2005.1552158

[8] MANDAL, B., CHATTERJEE. A., PARUI, S.K. Acrylic substrate based low profile wearable button antenna with FSS layer for WLAN and Wi-Fi applications. Microwave and Optical Technology Letters, 2015, vol. 57, no. 5, p. 1033-1038. DOI: 10.1002/mop.29012

[9] YANG, W., WANG, H., CHE, W. A Wideband and high-gain edge-fed patch antenna and array using artificial magnetic conductor structures. IEEE Antennas and Wireless Propagation Letters, 2013, vol. 12, p. 769-772. DOI: 10.1109/ LAWP.2013.2270943

[10] HERTL, I., RAIDA, Z., NOVACEK, Z. Multireflector antennas Cascaded structures with frequency selective surfaces. Radioengineering, 2006, vol. 15, no. 4, p. 80-83. ISSN 1210-2512

[11] BEHDAD, N. A second order band-pass frequency selective surface using non-resonant sub-wavelength periodic structures. Microwave and Optical Technology Letters, 2008, vol. 50, no. 6, p. 1639-1643. DOI: 10.1002/mop.23445

[12] AL-JOUMAYLY, M., BEHDAD, N. A new technique for design of low-profile, second-order, bandpass frequency selective surfaces. IEEE Transactions on Antennas and Propagation, 2009, vol. 57, no. 2, p. 452-459. DOI: 10.1109/TAP.2008.2011382

[13] CHATTERJEE, A., PARUI, S.K. A multi-layered band-pass frequency selective surface designed for $\mathrm{Ku}$ band applications. In IEEE Applied Electromagnetics Conference (AEMC). Bhubaneswar (India), 2013, p.1-2. DOI: 10.1109/AEMC.2013.7045067

[14] LUUKKONEN, O., SIMOVSKI, C., GRANET, G., GOUSSETIS, G., LIOUBTCHENKO, D., RAISANEN, A., TRETYAKIV, S Simple and accurate analytical model of planar grids and highimpedance surfaces comprising metal strips or patches. IEEE Transactions on Antennas and Propagation, 2008, vol. 56, no. 6, p. 1624-1632. DOI: 10.1109/TAP.2008.923327

[15] LANGLEY, R.J., PARKER, E.A. Equivalent circuit model for arrays of square loops. IET Electronics Letters, 1982, vol. 18, no. 7, p. 294-296. DOI: 10.1049/el:19820201

[16] YILMAZ, A.E., KUZUOGLU, M. Design of square loop frequency selective surfaces with particle swarm optimization via the equivalent circuit model. Radioengineering, 2009, vol. 18, no. 2 , p. 95-102. ISSN 1210-2512

[17] KENT, E.F., DOKEN, B., KARTAL, M. A new equivalent circuit based FSS design method by using genetic algorithm. In $2^{\text {nd }}$ International Conference on Engineering Optimization. Lisbon (Portugal), 2010, p. 1-4.

[18] BRAZ, E.C., CAMPOS, A.L.P.S. Dual/wide band multifractal frequency selective surface for applications in $\mathrm{S}$ and $\mathrm{X}$ band.
Microwave and Optical Technology Letters, 2014, vol. 56, no. 10, p. 2217-2222. DOI: $10.1002 / \mathrm{mop} .28561$

[19] LINS, H.W.C., BARRETO, E.L.F., D'ASSUNCAO, A.G. Enhanced wideband performance of coupled frequency selective surfaces using metaheuristics. Microwave and Optical Technology Letters, 2013, vol. 55, no. 4, p. 711-715. DOI: 10.1002/mop.27451

\section{About the Authors...}

Ayan CHATTERJEE (1988) received his B.Tech. degree in Electronics and Communication Engineering from West Bengal University of Technology, India in the year 2010. He did M.Tech. in Communication Engineering from University of Kalyani, India in 2012. From 2012 to 2013, he worked as an Assistant Professor in Supreme Knowledge Foundation Group of Institutions, West Bengal. Since 2013, he is pursuing Ph.D. from the Department of Electronics and Tele-Communication Engineering of the Indian Institute of Engineering Science \& Technology, Shibpur (formerly known as Bengal Engineering \& Science University). He was awarded Senior Research Fellowship by Council of Scientific and Industrial Research, India in the year 2014. His research interests include design of Frequency Selective Surfaces for radomes and antenna systems. He has publications in international journals and conference proceedings.

Susanta Kumar PARUI (1965) received his B.Sc. degree in Physics and B.Tech. degree in Radio Physics and Electronics from University of Calcutta in the year 1987 and 1990, respectively. He did M.E. and Ph.D in Microwave Engineering from Bengal Engineering and Science University (presently known as Indian Institute of Engineering Science \& Technology), Shibpur, India. From 1993 to 2000, he worked as an Instrument Engineer in Process control Industries. Since 2000, he is associated with the Department of Electronics and Tele-Communication Engineering of the Indian Institute of Engineering Science \& Technology, Shibpur and presently holds the post of Associate Professor. He was awarded post doctoral fellowship from Royal Academy of Engineering, U.K in the year 2009. Presently he is holding the position of Vice-Chairman, IEEE AP/MTT Chapter, Kolkata Section. His current research interests include planar circuits, antennas, SIW, DGS, EBG and Meta materials. He has published more than 50 contributory papers in referred journals and international conference proceedings. 